\title{
Effect of correlation on bond prices in short rate models of interest rates
}

\author{
Zuzana Girová, Beáta Stehíková
}

\begin{abstract}
Short rate models of interest rates are formulated in terms of stochastic differential equations which describe the evoution of an instantaneous interest rate, called short rate. Bonds and other interest rate derivatives are then priced by a parabolic partial differential equation. We consider two-factor models, in which also correlation between the factors enters the bond-pricing differential equation. Firstly, we study the dependence of the bond prices on the correlation in three particular short rate models. The differences and common features of the results motivate us to investigate the dependence of the solution to the bond-pricing partial differential equation on the parameter representing correlation between the factors in a general case.
\end{abstract}

\section{INTRODUCTION}

Short rate models of interest rates are formulated in terms of stochastic differential equations for the short rate. In this paper we consider twofactor models, in which there are two sources of uncertainity, modelled by two correlated Wiener processes. In particular, there are two random factors $x$ and $y$, which satisfy a system of stochastic differential equations

$$
\begin{aligned}
\mathbf{d} x= & \mu_{x}(x, y) \mathbf{d} t+\sigma_{x}(x, y) \mathbf{d} w_{1}, \\
\mathbf{d} y= & \mu_{y}(x, y) \mathbf{d} t+\sigma_{y}(x, y) \mathbf{d} w_{2}, \\
& \operatorname{cor}\left(\mathbf{d} w_{1}, \mathbf{d} w_{2}\right)=\rho,
\end{aligned}
$$

where we assume that $\mu_{x}, \mu_{y}, \sigma_{x}, \sigma_{y}$ do not depend explicitly on time and they satisfy condition to ensure the existence of stochastic processes $x$ and $y$, see [10] . The correlation $\rho \in(-1,1)$ is a constant. The short rate $r$ is a

2010 Mathematics Subject Classification. Primary: 35K15; Secondary: 35Q80.

Key words and phrases. Short rate model, bond price, correlation.

Full paper. Received 5 November 2018, revised 4 December 2018, accepted 9 December 2018, available online 15 December 2018.

The work was supported by VEGA 1/0251/16 grant and by Bilateral German-Slovak Project ENANEFA - Efficient Numerical Approximation of Nonlinear Equations in Financial Applications financed by DAAD and the Slovakian Ministry of Education. 
smooth function of the factors, i.e., $r=g(x, y)$. This framework nests many well known models; here we present some typical examples:

- In stochastic volatility models the short rate is one of the factors (i.e., $g(x, y)=x)$ and the other factor defines the instantaneouts volatility of the short rate (which translates into the choice $\sigma_{x}(x, y)=\sqrt{y}$ ). A particular stochastic volatility model proposed by Fong and Vasicek in [6] will be studied later in this paper; the reader is referred to [7] and [8] for a treatment of stochastic volatility models in finance and interest rate modelling in particular.

- Not only volatility, but also other variable influencing the short rate, which is again directly one of the factors, can be made stochastic and taken as the second factor. Examples include mean-reversion models with a stochastic mean and their special kind, a class of convergence models (see [4] for a basic model) where the short rate in the domestic country reverts to a short rate in a monetary union (that is itself stochastic and modelled as the second factor) which the country is going to join.

- Interest rate can be a sum of the two factors, i.e. $g(x, y)=x+y$. The factors might have a direct interpretation, for example in [11] the two factors correspond to the long rate and the spread (difference between thet short and long rates). In other cases, they may not correspond to a specific quantities; in [1] the are described as an influence of various economic news. The processes can have a form known from simpler one-factor models, such as [14] and [5], see for example [3].

We note that all of these cases, the correlation between the factors is one of the model parameters. Besides modelling the instantaneous interest rate, the model are used also to price the derivatives, i.e., financial instruments that have a value dependent on the interest rate. It follows that their prices depend on the correlation between the factors as well. In this paper we study the effect of the correlation on the prices of bonds. In particular, we derive the order of the difference between the logarithms of the bond prices for a zero correlation case and a case with a general value of the correlation. There are two reasons for choosing the logarithms. Firstly, it enables us to estimate the relative difference in the bond prices and secondly, it directly gives the difference in the implied interest rates, which are linked to bond prices by the formula $P=\exp (-R \times \tau)$, where $P$ is the bond price, $R$ is the corresponding interest rate and $\tau$ is time remaining to maturity of the bond. At the time of maturity (i.e., $\tau=0$ ), the bond prices are equal; however, it turns out that also higher order terms can be equal. We are therefore looking for such an $\alpha$ that the order of difference of the logarithms is $O\left(\tau^{\alpha}\right)$ as $\tau \rightarrow 0^{+}$. 
The paper is organized as follows: In Section 2 we describe the partial differential equations approach to pricing interest rate derivatives; in particular to pricing bonds. In Section 3 we present three specific examples of short rate models which differ by the form in which the solution to the bondpricing equation is known. We use appropriate techniques to determine in each case the order of difference of logarithms of the bond prices when computed for a general model and the for the case with zero correlation. The differences and similar features obtained in this way are a motivation for studying this question for a general two-factor model, which we do in Section 4. We conclude the paper in Section 5 where we summarize and discuss the results as well as their usefulness.

\section{Partial differential equations approach TO PRICING BONDS IN TWO-FACTOR SHORT RATE MODELS}

A derivative is a financial instrument whose value depends on an underlying asset or a financial quantity. In this paper we study bonds; they can be viewed as derivatives of the short rate (see for example [9] and [3] for more interest rate derivates). A zero-coupon discount bond is a security that pays a unit amount of money at a specified time $T$ called maturity of the bond. It is important in order to derive implied interest rates and also to discount future cash flows.

Suppose that the factors $x$ and $y$ evolve according to the system (1), (2), (3) and the short rate $r$ is given by a smooth function of the factors, $r=g(x, y)$. Then the price $P$ of a financial derivative depending on the short rate is a function $P(x, y, t)$ where $t$ is the current time. The two main approaches for computing $P$ are the partial differential equations approach which we will use here and risk-neutral approach where the prices are computed as discounted expected values under an equivalent (so called risk-neutral) probability measure. In the remaining part of this section we provide the basic result of partial differential equations pricing and we refer the reader to [9] for more details on both methods.

After a substitution $\tau=T-t$, where $\tau$ denotes time remaining to its maturity, the function $P(x, y, \tau)$ satisfies a parabolic partial differential equation

$$
\begin{aligned}
& -\frac{\partial P}{\partial \tau}+\left(\mu_{x}(x, y)-\sigma_{x}(x, y) \lambda_{x}(x, y)\right) \frac{\partial P}{\partial x} \\
& +\left(\mu_{y}(x, y)-\sigma_{y}(x, y) \lambda_{y}(x, y)\right) \frac{\partial P}{\partial y} \\
& +\frac{1}{2} \sigma_{x}(x, y)^{2} \frac{\partial^{2} P}{\partial x^{2}}+\frac{1}{2} \sigma_{y}(x, y)^{2} \frac{\partial^{2} P}{\partial y^{2}} \\
& +\rho \sigma_{x}(x, y) \sigma_{y}(x, y) \frac{\partial^{2} P}{\partial x \partial y}-g(x, y)=0
\end{aligned}
$$


for all admissible values of $x, y$ and for all $\tau \in(0, T)$. The functions $\lambda_{x}(x, y)$ and $\lambda_{y}(x, y)$ are so called market prices of risk and need to be specified in order to price derivatives, besides the stochastic differential formulation of the short rate evolution (1)-(3). In general they can depend also on time $t$ but have to be independent on maturity of the bond $T$. See [9] for a derivation of the partial differential equation in a two-factor interest rate model.

The initial condition $P(x, y, 0)$ is equal to the payoff of the derivative, therefore in case of a bond we have

$$
P(x, y, 0)=1
$$

for all $x$ and $y$, whose range depends on the specification of the processes.

\section{Dependence of the Bond PRICES}

ON THE CORRELATION IN SPECIFIC MODELS

In this section we consider three models which differ by the form in which the solution to the bond-pricing equation can be expressed. In two-factor Vasicek model the solution is simaple and it can be written in a closed form. In the case of Fong-Vasicek model we will work with the system of ordinary differential equations which characterizes functions arising in the separable form of the bond price. Finally, two-factor Cox-Ingersoll-Ross model has a closed form solution in the case of zero correlation, but in general it does not even have a separable solution and we only have the partial differential equation available. The first two cases were already presented at the Student Science Conference 2018 organized by Comenius University, Bratislava, and here we outline the results for reader's convenience.

3.1. Two-factor Vasicek model. In the two-factor Vasicek model (see [3]) the short rate is given as a sum of two factors $r_{1}$ and $r_{2}$, each of which is governed by an Ornestein-Uhlenbeck process, i.e., a mean-reverting stochastic differential equation with a constant volatility:

$$
\begin{aligned}
& \mathbf{d} r_{1}=\kappa_{1}\left(\theta_{1}-r_{1}\right) \mathbf{d} t+\sigma_{1} \mathbf{d} w_{1}, \\
& \mathbf{d} r_{2}=\kappa_{2}\left(\theta_{2}-r_{2}\right) \mathbf{d} t+\sigma_{2} \mathbf{d} w_{2},
\end{aligned}
$$

where $\kappa_{1}, \kappa_{2}, \sigma_{1}, \sigma_{2}>0$ and $\theta_{1}, \theta_{2} \in \mathbb{R}$ are parameters. We also denote the correlation between $\mathbf{d} w_{1}$ and $\mathbf{d} w_{2}$ by $\rho$. Considering constant market prices of risk $\lambda_{1}$ and $\lambda_{2}$, we can solve the corresponding bond-pricing partial differential equation (4) in a closed form. The solution is known to have a separable form

$$
P\left(r_{1}, r_{2}, \tau\right)=\exp \left(A(\tau)-B_{1}(\tau) r_{1}-B_{2}(\tau) r_{2}\right),
$$


where

$$
\begin{aligned}
\dot{A}= & -\left(\kappa_{1} \theta_{1}-\lambda_{1} \sigma_{1}\right) B_{1}-\left(\kappa_{2} \theta_{2}-\lambda_{2} \sigma_{2}\right) B_{2} \\
& +\frac{1}{2} \sigma_{1}^{2} B_{1}^{2}+\frac{1}{2} \sigma_{2}^{2} B_{2}^{2}+\rho \sigma_{1} \sigma_{2} B_{1} B_{2}, \\
\dot{B}_{1}= & 1-\kappa_{1} B_{1}, \\
\dot{C}_{2}= & 1-\kappa_{2} B_{2}
\end{aligned}
$$

and $A(0)=B(0)=C(0)$.

This system of equations can be easily solved and using its closed form solution the difference between the logarithms of bond prices with correlation $\rho=0$ and a general correlation $\rho \in(-1,1)$ can be expressed and expanded into a Taylor serie around $\tau=0$ :

$$
\begin{aligned}
& \log P\left(r_{1}, r_{2}, \tau ; 0\right)-\log P\left(r_{1}, r_{2}, \tau ; \rho\right) \\
& =-\rho \frac{\sigma_{1} \sigma_{2}}{\kappa_{1} \kappa_{2}}\left(\tau-\frac{1-e^{-\kappa_{1} \tau}}{\kappa_{1}}-\frac{1-e^{-\kappa_{2} \tau}}{\kappa_{2}}+\frac{1-e^{-\left(\kappa_{1}+\kappa_{2}\right) \tau}}{\kappa_{1}+\kappa_{2}}\right) \\
& =-\frac{1}{3} \rho \sigma_{1} \sigma_{2} \tau^{3}+O\left(\tau^{4}\right) .
\end{aligned}
$$

3.2. Fong-Vasicek model with stochastic volatility. The Fong-Vasicek model [6] assumes that the short rate $r$ follows a mean-reverting process similar to one-factor Vasicek case [14], but instead of a constant volatility, its second power (i.e., the instanteneous variance) is modelled by a Bessel square root process:

$$
\begin{aligned}
& \mathbf{d} r=\kappa_{1}\left(\theta_{1}-r_{1}\right) \mathbf{d} t+\sqrt{y} \mathbf{d} w_{1}, \\
& \mathbf{d} y=\kappa_{2}\left(\theta_{2}-y\right) \mathbf{d} t+v \sqrt{y} \mathbf{d} w_{2},
\end{aligned}
$$

where $\kappa_{1}, \kappa_{2}, \theta_{1}, \theta_{2}, \sigma_{1}, \sigma_{2}>0$ are parameters. Again, we denote the correlation between $\mathbf{d} w_{1}$ and $\mathbf{d} w_{2}$ by $\rho$. Market prices of risk are assumed to be proportional to $\sqrt{y}$, we denote then by $\lambda_{1} \sqrt{y}$ and $\lambda_{2} \sqrt{y}$ respectively.

In this setting, the bond price has again a separable solution in the form

$$
P(r, y, \tau)=\exp (A(\tau)-B(\tau) r-C(\tau) y),
$$

where the functions $A, B, C$ are characterized by a system of ordinary differential equations (see, for example [13])

$$
\begin{aligned}
& \dot{A}=-\kappa_{1} \theta_{1} B-\kappa_{2} \theta_{2} C \\
& \dot{B}=-\kappa_{1} B+1 \\
& \dot{C}=-\lambda_{1} B-\kappa_{2} C-\lambda_{2} v C-\frac{1}{2} B^{2}-\frac{1}{2} v^{2} C^{2}-v \rho B C
\end{aligned}
$$

with initial conditions $A(0)=B(0)=C(0)=0$. We remark that the solution can be expressed in a serie form (see [12]), but the system of equations 
will be a convenient tool for our derivation of the dependence of bond prices on the correlation $\rho$. In particular, we have

$$
\begin{aligned}
\log P(r, y, \tau ; 0)-\log P(r, y, \tau ; \rho)= & -\kappa_{2} \theta_{2} \int_{0}^{\tau}\left[C(s)-C_{0}(s)\right] \mathbf{d} s \\
& -\left[C(\tau)-C_{0}(\tau)\right] y,
\end{aligned}
$$

where we denoted by $C_{0}$ the function $C$ for the case of $\rho=0$. Now, we can compute the Taylor expansion of the function $C$ using the system of equations (7)-(9) and we obtain

$$
\log P(r, y, \tau ; 0)-\log P(r, y, \tau ; \rho)=\frac{1}{8} \rho v \lambda_{1} y \tau^{4}+O\left(\tau^{5}\right) .
$$

At this place we note that for the Fong-Vasicek model, the difference is one order higher compared to the two-factor Vasicek model considered in the previous subsection.

3.3. Two-factor Cox-Ingersoll-Ross model. In an analogous manner as the two-factor Vasicek model, the two-factor Cox-Ingersoll-Ross model (see [3], CIR hereafter) is formulated. The short rate $r$ is a sum of two processes, each of which follows a stochastic differential equation known from the onefactor CIR model [5], which is a Bessel square root process:

$$
\begin{aligned}
& \mathbf{d} r_{1}=\kappa_{1}\left(\theta_{1}-r_{1}\right) \mathbf{d} t+\sigma_{1} \mathbf{d} w_{1}, \\
& \mathbf{d} r_{2}=\kappa_{2}\left(\theta_{2}-r_{2}\right) \mathbf{d} t+\sigma_{2} \mathbf{d} w_{2},
\end{aligned}
$$

where $\kappa_{1}, \kappa_{2}, \theta_{1}, \theta_{2}, \sigma_{1}, \sigma_{2}>0$ are parameters. Again, we denote the correlation between $\mathbf{d} w_{1}$ and $\mathbf{d} w_{2}$ by $\rho$. Market prices of risk are assumed to be proportional to each of the factors and we denote then by $\lambda_{1} \sqrt{r_{1}}$ and $\lambda_{2} \sqrt{r_{2}}$ respectively.

For this model a separable solution of the form (6) exists only in the case of $\rho=0$, when it can be expressed in closed form. For a convenience we write the system of ordinary differential equations, from which the solution arises:

$$
\begin{aligned}
& \dot{A}=-\kappa_{1} \theta_{1} B_{1}-\kappa_{2} \theta_{2} B_{2}, \\
& \dot{B}_{1}=1-\left(\kappa_{1}+\lambda_{1} \sigma_{1}\right) B_{1}-\frac{\sigma_{1}^{2}}{2} B_{1}^{2}, \\
& \dot{B}_{2}=1-\left(\kappa_{2}+\lambda_{2} \sigma_{2}\right) B_{2}-\frac{\sigma_{2}^{2}}{2} B_{2}^{2},
\end{aligned}
$$

with initial conditions $A(0)=B_{1}(0)=B_{2}(0)=0$. However, to evaluate the bond prices when $\rho \neq 0$ we need to solve the partial differential equation for $P\left(r_{1}, r_{2}, \tau\right)$ numerically (or employ another approximation technique, such as a Monte Carlo simulation). If the effect of correlation is small, we can approximate (maybe as a first approximation for example in the first stage of a calibration) this price by the closed form solution obtained in the uncorrelated case $\rho=0$. 
In order to obtain the estimates similar to previous section, we refer to the bond-pricing partial differential equation satisfied by the exact bond prices. Since we are interested in difference of logarithms, we firstly transform the bond-pricing equation into an equation for the logarithm of the bond price. If we denote $f\left(r_{1}, r_{2}, \tau\right)=\log P\left(r_{1}, r_{2}, \tau ; \rho\right)$, then we have

$$
\begin{aligned}
& -\frac{\partial f}{\partial \tau}+\left(\kappa_{1}\left(\theta_{1}-r_{1}\right)-\lambda_{1} \sigma_{1} r_{1}\right) \frac{\partial f}{\partial r_{1}}+\left(\kappa_{2}\left(\theta_{2}-r_{2}\right)-\lambda_{2} \sigma_{2} r_{2}\right) \frac{\partial f}{\partial r_{2}} \\
& +\frac{1}{2} \sigma_{1}^{2} r_{1}\left[\left(\frac{\partial f}{\partial r_{1}}\right)^{2}+\frac{\partial^{2} f}{\partial r_{1}^{2}}\right]+\frac{1}{2} \sigma_{2}^{2} r_{2}\left[\left(\frac{\partial f}{\partial r_{2}}\right)^{2}+\frac{\partial^{2} f}{\partial r_{2}^{2}}\right] \\
& +\rho \sigma_{1} \sigma_{2} \sqrt{r_{1} r_{2}}\left[\frac{\partial^{2} f}{\partial r_{1} \partial r_{2}}+\frac{\partial f}{\partial r_{1}} \frac{\partial f}{\partial r_{2}}\right]-\left(r_{1}+r_{2}\right)=0
\end{aligned}
$$

If, instead of the exact solution $f$, we substitute

$$
f_{0}\left(r_{1}, r_{2}, \tau\right)=\log P\left(r_{1}, r_{2}, \tau ; 0\right)
$$

into the left hand side, we obtain a nontrivial right hand side, which we denote by $h\left(r_{1}, r_{2}, \tau\right)$. In particular, we have

$$
h\left(r_{1}, r_{2}, \tau\right)=\rho \sigma_{1} \sigma_{2} \sqrt{r_{1} r_{2}} B_{1}(\tau) B_{2}(\tau),
$$

where $B_{1}$ and $B_{2}$ are components of the separable solution in the uncorrelated case given by (10)-(11). Therefore $B_{1}(\tau) B_{2}(\tau)=\tau^{2}+O\left(\tau^{2}\right)$ and

$$
h\left(r_{1}, r_{2}, \tau\right)=\rho \sigma_{1} \sigma_{2} \sqrt{r_{1} r_{2}} \tau^{2}+O\left(\tau^{3}\right) .
$$

Next, we define

$$
g\left(r_{1}, r_{2}, \tau\right)=\log P\left(r_{1}, r_{2}, \tau ; 0\right)-\log P\left(r_{1}, r_{2}, \tau ; \rho\right)
$$

and write the partial differential equation which the function $g$ satisfies:

$$
\begin{aligned}
& -\frac{\partial g}{\partial \tau}+\left(\kappa_{1}\left(\theta_{1}-r_{1}\right)-\lambda_{1} \sigma_{1} r_{1}\right) \frac{\partial g}{\partial r_{1}}+\left(\kappa_{2}\left(\theta_{2}-r_{2}\right)-\lambda_{2} \sigma_{2} r_{2}\right) \frac{\partial g}{\partial r_{2}} \\
+ & \frac{1}{2} \sigma_{1}^{2} r_{1}\left[\left(\frac{\partial g}{\partial r_{1}}\right)^{2}+\frac{\partial^{2} g}{\partial r_{1}^{2}}\right]+\frac{1}{2} \sigma_{2}^{2} r_{2}\left[\left(\frac{\partial g}{\partial r_{2}}\right)^{2}+\frac{\partial^{2} g}{\partial r_{2}^{2}}\right] \\
+ & \rho \sigma_{1} \sigma_{2} \sqrt{r_{1} r_{2}}\left[\frac{\partial^{2} g}{\partial r_{1} \partial r_{2}}+\frac{\partial g}{\partial r_{1}} \frac{\partial g}{\partial r_{2}}\right] \\
= & h\left(r_{1}, r_{2}, \tau\right)+\frac{1}{2} \sigma_{1}^{2} r_{1}\left[\left(\frac{\partial f}{\partial r_{1}}\right)^{2}-\frac{\partial f_{0}}{\partial r_{1}} \frac{\partial f}{\partial r_{1}}\right] \\
& +\frac{1}{2} \sigma_{2}^{2} r_{2}\left[\left(\frac{\partial f}{\partial r_{2}}\right)^{2}-\frac{\partial f_{0}}{\partial r_{2}} \frac{\partial f}{\partial r_{2}}\right] \\
& \cdot \rho \sigma_{1} \sigma_{2} \sqrt{r_{1} r_{2}}\left[2 \frac{\partial f}{\partial r_{1}} \frac{\partial f}{\partial r_{2}}-\frac{\partial f_{0}}{\partial r_{1}} \frac{\partial f}{\partial r_{2}}-\frac{\partial f}{\partial r_{1}} \frac{\partial f_{0}}{\partial r_{2}}\right]
\end{aligned}
$$


If we write the function $g$ in its Taylor serie form

$$
g\left(r_{1}, r_{2}, \tau\right)=\sum_{k=0}^{\infty} c_{k}\left(r_{1}, r_{2}\right) \tau^{k}=\sum_{k=k_{0}}^{\infty} c_{k}\left(r_{1}, r_{2}\right) \tau^{k},
$$

then we know that $k_{0}>0$ because both $f$ and $f_{0}$ are zero for $\tau=0$ as a consequence of the terminal condition (5) for the bond price in the general bond-pricing partial differential equation (4). Therefore, the left hand side of (12) is of order $O\left(\tau^{k_{0}-1}\right)$. Using the fact that $f$ and $f_{0}$ are both $O(\tau)$ and $h$ is $O\left(\tau^{2}\right)$ we conclude that the right hand side of (12) is $O\left(\tau^{2}\right)$. Therefore $k_{0} \geq 3$ and we able to make more precise estimates of the terms on the right hand side of (12):

$$
\left(\frac{\partial f}{\partial r_{1}}\right)^{2}-\frac{\partial f_{0}}{\partial r_{1}} \frac{\partial f}{\partial r_{1}}=\frac{\partial f}{\partial r_{1}}\left(\frac{\partial f}{\partial r_{1}}-\frac{\partial f_{0}}{\partial r_{1}}\right)=O(\tau) \times O\left(\tau^{3}\right)=O\left(\tau^{4}\right)
$$

and similarly for $r_{2}$. Also,

$$
\begin{aligned}
& 2 \frac{\partial f}{\partial r_{1}} \frac{\partial f}{\partial r_{2}}-\frac{\partial f_{0}}{\partial r_{1}} \frac{\partial f}{\partial r_{2}}-\frac{\partial f}{\partial r_{1}} \frac{\partial f_{0}}{\partial r_{2}} \\
= & \frac{\partial f}{\partial r_{1}}\left(\frac{\partial f}{\partial r_{2}}-\frac{\partial f_{0}}{\partial r_{2}}\right)+\frac{\partial f}{\partial r_{2}}\left(\frac{\partial f}{\partial r_{1}}-\frac{\partial f_{0}}{\partial r_{1}}\right) \\
= & O(\tau) \times O\left(\tau^{3}\right)+O(\tau) \times O\left(\tau^{3}\right)=O\left(\tau^{4}\right)
\end{aligned}
$$

and therefore the only $O\left(\tau^{2}\right)$ term from the right hand side of (12) comes from the function $h$ and it is equal to $\rho \sigma_{1} \sigma_{2} \sqrt{r_{1} r_{2}} \tau^{2}$. Finally, we are able to conclude that $k_{0}=3$ and

$$
\begin{aligned}
g\left(r_{1}, r_{2}, \tau\right) & =\log P\left(r_{1}, r_{2}, \tau ; 0\right)-\log P\left(r_{1}, r_{2}, \tau ; \rho\right) \\
& =-\frac{1}{3} \sigma_{1} \sigma_{2} \sqrt{r_{1} r_{2}} \tau^{3}+O\left(\tau^{3}\right) .
\end{aligned}
$$

This order of accuracy is the same as in the two-factor Vasicek model. Moreover, the leading term has a similar form; instead of constant volatilities in the Vasicek model it has instantaneous volatilities from the CIR model formulation.

\section{Dependence on Correlation in a general two-factor model}

The three examples from the previous section were a motivation for studying the effect of correlation in a general two-factor short rate model. Therefore, in this section we consider a general model described in the introduction, when the short rate $r$ is given as a certain smooth function of the auxiliary processes $x$ and $y$ governed by (1) and (2) respectively, i.e. $r=g(x, y)$. We derive the order of difference in logarithms of the bond prices computed for $\rho=0$ and a general nonzero value of the correlation $\rho$. In particular, we distinguish the cases $g(x, y)=x$ and $g(x, y)=x+y$ and remark on some 
other illustrative choices of $g$, but the analysis can be performed for any function $g$.

To simplify the notation, in what follows we denote $\tilde{\mu}_{i}(x, y)=\mu_{i}(x, y)-$ $\lambda_{i}(x, y) \sigma_{i}(x, y)$ for $i \in\{x, y\}$. Then, the partial differential equation for $f(x, y, \tau)=\log P(x, y, \tau)$ reads as

$$
\begin{aligned}
& -\frac{\partial f}{\partial \tau}+\tilde{\mu}_{x}(x, y) \frac{\partial f}{\partial x}+\tilde{\mu}_{y}(x, y) \frac{\partial f}{\partial y} \\
& +\frac{1}{2} \sigma_{x}(x, y)^{2}\left[\left(\frac{\partial f}{\partial x}\right)^{2}+\frac{\partial^{2} f}{\partial x^{2}}\right]+\frac{1}{2} \sigma_{y}(x, y)^{2}\left[\left(\frac{\partial f}{\partial y}\right)^{2}+\frac{\partial^{2} f}{\partial y^{2}}\right] \\
& +\rho \sigma_{x}(x, y) \sigma_{y}(x, y)\left[\frac{\partial^{2} f}{\partial x \partial y}+\frac{\partial f}{\partial x} \frac{\partial f}{\partial y}\right]-g(x, y)=0
\end{aligned}
$$

We write $f$ in its Taylor expansion form

$$
f(x, y, \tau)=\sum_{k=0}^{\infty} c_{k}(x, y) \tau^{k}
$$

and note that $c_{0}(x, y)$ is identically equal to zero because of the terminal condition (5) for the bond price in the general bond-pricing partial differential equation (4). To find the order of the difference $f(x, y, \tau ; 0)-f(x, y, \tau, \rho)$ we need to find the first term $c_{k}$ which depends on $\rho$. Substituting the Taylor expansion in the partial differential equation for $f$ we get

$$
c_{1}(x, y)=-g(x, y)
$$

and

$$
\begin{aligned}
c_{2}(x, y)= & \frac{1}{2}\left[\tilde{\mu}_{x}(x, y) \frac{\partial c_{1}}{\partial x}+\tilde{\mu}_{y}(x, y) \frac{\partial c_{1}}{\partial y}+\frac{1}{2} \sigma_{x}(x, y)^{2} \frac{\partial^{2} c_{1}}{\partial x^{2}}\right. \\
& \left.+\frac{1}{2} \sigma_{y}(x, y)^{2} \frac{\partial^{2} c_{1}}{\partial y^{2}}+\rho \sigma_{x}(x, y) \sigma_{y}(x, y) \frac{\partial^{2} c_{1}}{\partial x \partial y}\right] .
\end{aligned}
$$

This term depends on $\rho$ if the second partial derivative $\partial^{2} g / \partial x \partial y$ is nonzero. We can recall a one-factor Black-Karasinski model [2] where the short rate is defined as $r=\exp (x)$ where $x$ is an Ornstein-Uhlenbeck process. If we define the short rate in an analogous way in a two-factor model as $r=\exp (x+y)$ for some processes $x$ and $y$, then the difference $f(x, y, \tau ; 0)-f(x, y, \tau, \rho)$ is only $O\left(\tau^{2}\right)$; in particular we can write in terms of the function $g$ that

$$
f(x, y, \tau ; 0)-f(x, y, \tau, \rho)=\frac{1}{2} \rho \sigma_{x}(x, y) \sigma_{y}(x, y) \frac{\partial^{2} g}{\partial x \partial y} \tau^{2}+O\left(\tau^{2}\right)
$$

If this is not the case and the derivative $\partial^{2} g / \partial x \partial y$ is zero, it means that the function $g$ has a separable form $g(x, y)=g_{1}(x)+g_{2}(y)$ for some functions of one variable $g_{1}$ and $g_{2}$. In this case we compute the next term of the Taylor 
expansion:

$$
\begin{aligned}
c_{3}(x, y)= & \frac{1}{3}\left[\tilde{\mu}_{x}(x, y) \frac{\partial c_{2}}{\partial x}+\tilde{\mu}_{y}(x, y) \frac{\partial c_{2}}{\partial y}+\frac{1}{2} \sigma_{x}(x, y)^{2}\left(\frac{\partial^{2} c_{2}}{\partial x^{2}}+\left(\frac{\partial c_{1}}{\partial x}\right)^{2}\right)\right. \\
& +\frac{1}{2} \sigma_{y}(x, y)^{2}\left(\frac{\partial^{2} c_{2}}{\partial y^{2}}+\left(\frac{\partial c_{1}}{\partial y}\right)^{2}\right) \\
& \left.+\rho \sigma_{x}(x, y) \sigma_{y}(x, y)\left(\frac{\partial^{2} c_{2}}{\partial x \partial y}+\frac{\partial c_{1}}{\partial x} \frac{\partial c_{1}}{\partial y}\right)\right] .
\end{aligned}
$$

By taking derivatives of $c_{1}$ and $c_{2}$, this expression can be written in terms of the function $g$, risk neutral drifts and volatilities only, but we omit this expression for the sake of brevity. If the dependence of $c_{3}$ on $\rho$ does not vanish, then the difference $f(x, y, \tau ; 0)-f(x, y, \tau, \rho)$ is $O\left(\tau^{3}\right)$; in particular

$$
\begin{aligned}
& f(x, y, \tau ; 0)-f(x, y, \tau, \rho) \\
= & -\frac{1}{3} \sigma_{x}(x, y) \sigma_{y}\left(x, y\left(\frac{\partial^{2} c_{2}}{\partial x \partial y}+\frac{\partial c_{1}}{\partial x} \frac{\partial c_{1}}{\partial y}\right) \tau^{3}+O\left(\tau^{3}\right) .\right.
\end{aligned}
$$

Remark 4.1. Since both models from the previous section which had $g$ given by $g(x, y)=x+y$ fell into this cathegory, we find out now, if it is possible to achieve a higher order for this choice of $g$ with different processes $x$ and $y$.

Firstly we note that in this case the term $c_{2}$ simplifies to

$$
c_{2}(x, y)=-\frac{1}{2}\left(\tilde{\mu}_{x}(x, y)+\tilde{\mu}_{y}(x, y)\right)
$$

and therefore

$$
\frac{\partial^{2} c_{2}}{\partial x \partial y}+\frac{\partial c_{1}}{\partial x} \frac{\partial c_{1}}{\partial y}=-\frac{1}{2} \frac{\partial}{\partial x \partial y}\left(\tilde{\mu}_{x}(x, y)+\tilde{\mu}_{y}(x, y)\right)+1 .
$$

This term vanishes only if the sum of risk-neutral drifts of the processes $x$ and $y$ has the form

$$
\tilde{\mu}_{x}(x, y)+\tilde{\mu}_{y}(x, y)=2 x y+F(x)+G(y),
$$

where $F$ and $G$ are some functions of one variable. Although we could theoretically construct such processes, the term $2 x y$ (which is the only one that "combines" variables $x$ and $y$ ) does not leave any space for choosing parameters that could be fit into real data and therefore it would be only a theoretical example.

Remark 4.2. In the previous section we encountered one model for which the studied order was higher than $O\left(\tau^{3}\right)$. It was the Fong-Vasicek model with $g(x, y)=x$, i.e., the short rate is directly one of the factors, for which the stochastic differential equations are formulated. Now we investigate the question, what additional conditions need to be added so that we obtain a higher order than $O\left(\tau^{3}\right)$. 
If $g(x, y)=x$, then the term $c_{2}$ has a simple form

$$
c_{2}(x, y)=-\frac{1}{2} \tilde{\mu}_{x}(x, y)
$$

and therefore

$$
\frac{\partial^{2} c_{2}}{\partial x \partial y}+\frac{\partial c_{1}}{\partial x} \frac{\partial c_{1}}{\partial y}=-\frac{1}{2} \frac{\partial \tilde{\mu}_{x}(x, y)}{\partial x \partial y} .
$$

Therefore the risk neutral drift of the short rate needs to have the separated form

$$
\tilde{\mu}_{x}(x, y)=F(x)+G(y)
$$

where $F$ and $G$ are functions of one variable. Note that this was the case also of the Fong-Vasicek model, where (we write $x$ instead of $r$ to keep up with the notation of our derivation)

$$
\tilde{\mu}_{x}(x, y)=\kappa_{1}\left(\theta_{1}-x\right)-\sqrt{y} \times \lambda_{1} \sqrt{y}=\kappa_{1} \theta_{1}-\kappa_{1} x-\lambda_{1} y
$$

has indeed a separable form.

Returning to our analysis of the general case, we finally evaluate the term $c_{4}$ as

$$
\begin{aligned}
c_{4}(x, y)= & \frac{1}{4}\left[\tilde{\mu}_{x}(x, y) \frac{\partial c_{3}}{\partial x}+\tilde{\mu}_{y}(x, y) \frac{\partial c_{3}}{\partial y}+\frac{1}{2} \sigma_{x}(x, y)^{2}\left(\frac{\partial^{2} c_{3}}{\partial x^{2}}+2 \frac{\partial c_{1}}{\partial x} \frac{\partial c_{2}}{\partial x}\right)\right. \\
& +\frac{1}{2} \sigma_{y}(x, y)^{2}\left(\frac{\partial^{2} c_{3}}{\partial y^{2}}+2 \frac{\partial c_{1}}{\partial y} \frac{\partial c_{2}}{\partial y}\right) \\
& \left.+\rho \sigma_{x}(x, y) \sigma_{y}(x, y)\left(\frac{\partial^{2} c_{3}}{\partial x \partial y}+\frac{\partial c_{1}}{\partial x} \frac{\partial c_{2}}{\partial y}+\frac{\partial c_{2}}{\partial x} \frac{\partial c_{1}}{\partial y}\right)\right] .
\end{aligned}
$$

and obtain the difference $f(x, y, \tau ; 0)-f(x, y, \tau, \rho)$ of order $O\left(\tau^{4}\right)$ with leading term

$$
\begin{aligned}
& f(x, y, \tau ; 0)-f(x, y, \tau, \rho) \\
= & -\frac{1}{4} \rho \sigma_{x}(x, y) \sigma_{y}(x, y)\left(\frac{\partial^{2} c_{3}}{\partial x \partial y}+\frac{\partial c_{1}}{\partial x} \frac{\partial c_{2}}{\partial y}+\frac{\partial c_{2}}{\partial x} \frac{\partial c_{1}}{\partial y}\right) \tau^{4}+O\left(\tau^{4}\right),
\end{aligned}
$$

which encompasses also the case of the Fong-Vasicek model from the previous section, for which this term does not vanish.

\section{Conclusion}

Motivated by three particular models (two factor Vasicek model, FongVasicek model with stochastic volatility, two factor Cox-Ingersoll-Ross model) we studied the dependence of bond prices on the correlation $\rho$ between the factors defining the short rate. In a general two factor model considered we have the short rater as a function of two factors $x$ and $y$, i.e., $r=g(x, y)$. We derived order of the difference between logarithms of the bond prices computed with $\rho=0$ and general $\rho$ as $\tau \rightarrow 0^{+}$and observed conditions on the model which lead to different orders of difference. 
We can highlight several interesting points: If the function $g(x, y)$ does not have a separable form $g(x, y)=g_{1}(x)+g_{2}(y)$, then the difference is only $O\left(\tau^{2}\right)$. In the separable form, the order of at least $O\left(\tau^{3}\right)$ is guaranteed. If the short rate is a sum of the factors, in a generic case the order $O\left(\tau^{3}\right)$ is attained (a condition for a higher order has been derived, but it does not lead to any well known short rate model). If the short rate is directly one of the factors, then a condition which guarantees a higher order than $O\left(\tau^{3}\right)$ (in a generic case the $O\left(\tau^{4}\right)$ order is obtained) is linearity of the risk neutral drift of the short rate process.

In some cases, the bond price with a zero correlation may be easier to compute or it might have a form leading to easier calibration. Our results show that if the maturity of the bond is not large, we can approximate the bond price with its countepart computed for a zero correlation. They also show, in which cases we can expect to obtain a better approximation in this way.

\section{REFERENCES}

[1] A. H. Babbs, K. B. Nowman, Kalman filtering of generalized Vasicek term structure models, J. Finan. Quant. Anal., 34 (1) (1999), 115-130.

[2] F. Black, P. Karasinski,. Bond and option pricing when short rates are lognormal, Financ. Anal. J., 47 (4) (1991), 52-59.

[3] D. Brigo, F. Mercurio, Interest rate models - theory and practice: With smile, inflation and credit, Springer, Berlin, 2006.

[4] T. Corzo Santamaria, E. S. Schwartz, Convergence within the EU: Evidence from interest rates, Econ. Notes, 29 (2) (2000), pp. 243-266.

[5] J. C. Cox, J. E. Ingersoll, S. A. Ross, A theory of the term structure of interest rates, Econometrica, 53 (2) (1985), 385-407.

[6] H. G. Fong, O. A. Vasicek, Fixed-income volatility management, J. Portfol. Manage., 17 (4) (1991), 41-46.

[7] J. P. Fouque, G. Papanicolaou, K. R. Sircar, Derivatives in financial markets with stochastic volatility, Cambridge University Press, Cambridge, 2000.

[8] J. P. Fouque, G. Papanicolaou, R. Sircar, K. Solna, Multiscale stochastic volatility for equity, interest rate, and credit derivatives, Cambridge University Press, Cambridge, 2011.

[9] Y. K. Kwok, Mathematical models of financial derivatives, Springer, Berlin, 2008.

[10] B. Oksendal, Stochastic differential equations, Springer, Berlin, 2003.

[11] S. M. Schaefer, E. S. Schwartz, A two-factor model of the term structure: An approximate analytical solution, J. Finan. Quant. Anal., 19 (4) (1984), 413-424.

[12] M. Selby, C. Strickland, Computing the Fong and Vasicek Pure Discount Bond Price Formula, J. Fixed Income, 5 (2) (1995), 78-84. 
[13] B. Stehlíková, D. Ševčovič, On a volatility averaging in a two-factor interest rate model, Proceedings of Algoritmy 2005, 17th Conference on Scientific Computing, Vysoké Tatry - Podbanské, Slovakia, 325-333.

[14] O. Vasicek, An equilibrium characterization of the term structure, J. Finan. Econ., 5 (2) (1977), 177-188.

\section{Zuzana Girová}

Faculty of Mathematics, Physics and Informatics

Comenius Universlit

MLYNSKÁ DOLINA

84248 BRATISLAVA

SLOVAKIA

E-mail address: z.girova@gmail.com

\section{BeÁta Stehíková}

Faculty of Mathematics, Physics and Informatics

Comenius Universlity

MLYNSKÁ DOLINA

84248 BRATISLAVA

SLOVAKIA

E-mail address: stehlikova@fmph.uniba.sk 